\title{
Economical rapid-prototyping of aspherical lenses
}

\author{
Angelina Müller ${ }^{1}$, Matthias C. Wapler ${ }^{1}$, and Ulrike Wallrabe ${ }^{1, *}$ \\ ${ }^{1}$ University of Freiburg, IMTEK - Department of Microsystems Engineering, Laboratory for Microactuators, Freiburg, Germany
}

\begin{abstract}
We present a rapid-prototyping process to fabricate aspherical lens arrays based on surface deformation due to thermal expansion of PDMS. Using laser-structuring and molding in combination with an FEM-based shape optimization, we were able to design, fabricate and characterize different micro-lens arrays. This fabrication process can be used for almost any kind of arbitrary lens shape, which allows for a large design freedom for micro lenses.
\end{abstract}

\section{Introduction}

Freeform micro lenses and micro lens arrays have many applications, e.g. in imaging systems, 3D displays, optical sensors or light sources [1]. Cost, design freedom and quality are the essential key figures. Some manufacturing methods have the disadvantage of being very expensive and time-consuming, e.g., precision machining technologies, two-photon polymerization techniques or grayscale lithography. Other processes including inkjet printing or reflow processes have limitations in the lens geometry. Our rapid-prototyping process, however, can produce nearly any lens shape, allowing a high degree of freedom in the design of micro-optical lenses. In particular, it is cost-effective also for small quantities.

\section{Design and fabrication}

Our fabrication method is based on the surface deformation due to thermal expansion of a soft polymer, polydimethylsiloxane (PDMS), in a cavity, first described by Brunne et al. [2]. The fabrication consist of two molding steps as illustrated in Fig 1. First, we laser-structure the initial cavity into a thick silicon substrate. Then, we fill liquid PDMS (Sylgard 182 from DowSil) into the cavity, cover it with a silane-coated glass slide and cure it in a mechanical press at a pressure of approx. $10 \mathrm{MPa}$ and a temperature of $120^{\circ} \mathrm{C}$. Before curing, the liquid PDMS expands during heating, such that excess material escapes from the cavity via spacers (see image of step (2)). After approx. 40 min the PDMS is polymerized, we remove the slide and the material shrinks during cooling. This results in a small but smooth surface deformation, which is generally dependent on the depth and shape of the initial cavity, the polymerization shrinkage, the coefficient of thermal expansion and the Poisson's ratio. The deformed surface acts as a mold in the second molding step where we fill the mold with a UV-curing adhesive, cover it with a glass substrate and cured it with UV exposure. We were able to determine the shape of the initial cavity for almost any lens shape using an FEM shape optimization, which needs the desired PDMS curing temperature and material
(1)

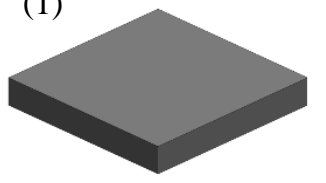

(3)

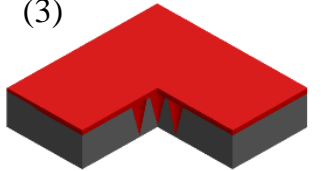

(5)

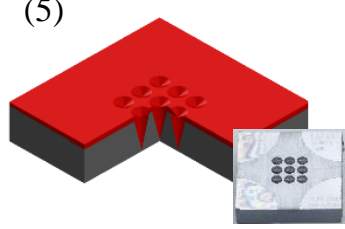

(7)

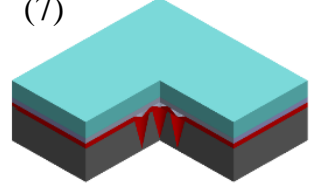

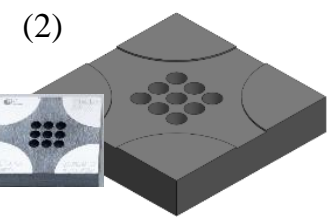

(4)

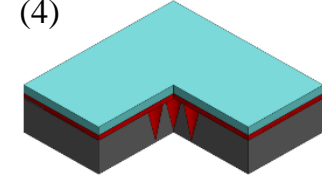

(6)

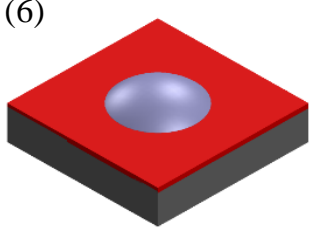

(8)

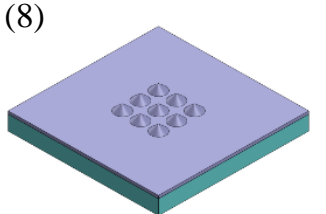

(9)

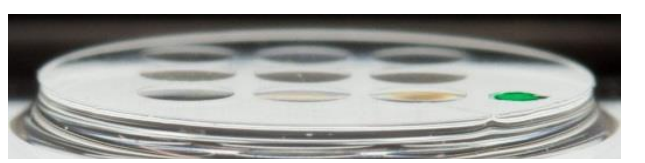

Fig. 1. Fabrication process of the transmissive aspherical optics. A funnel-shaped cavity is laser-structured into a thick silicon substrate (1 and 2). The uncured liquid PDMS is filled into the cavity (3), sealed with a glass slide (4) and heated under pressure. The still liquid polymer expands due to the elevated temperature and is pressed out of the mold. After polymerization, the device is cooled down, and the cured polymer shrinks. Small surface deformations form the lens molds (5). A UV-curable adhesive is dispensed on top of the lens molds (6), and a glass slide is pressed on (7). The device is then cured with UV-exposure, and the glass slide with the cured UV-adhesive is removed (8).

\footnotetext{
Corresponding author: wallrabe@imtek.uni-freiburg.de
} 
parameters. For this purpose, we have recently developed a new, accurate and quick method to determine the Poisson's ratio and the coefficient of thermal expansion of PDMS [3].

\section{Characterization and results}

We demonstrate our process with a $3 \times 3$ microlens (axicon) array with an element diameter of $1 \mathrm{~mm}$ and a conical lens shape with $174^{\circ}$ apex angle.

For the characterization of our lens array as shown in Fig. 2, we measured the surface quality of each lens with a high-precision optical profilometer. We determined the deviation of the lens shape from the intended shape (simulated target function $f_{\text {target }}$ ) for three different positions (Fig. 3), where we can observe smaller deviations. Lens P5 deviates strongly from the intended lens shape in the center, which suggests some particle, air bubble or void in the PDMS during the fabrication process. Furthermore, we determined the surface error of the three lens positions as a deviation from rotational symmetry and found a surface error of $113 \pm 13 \mathrm{~nm}$ (Tab. 1). In addition to the surface measurement, we verified the optical performance by illuminating the axicons with a collimated laser beam of $\lambda=473 \mathrm{~nm}$ and measured the resulting beam profile. In Fig. 4, we see a very good quality of the Bessel beam along with little distortion as expected from [4] with a reconstructed wavefront error of around $\lambda / 6$.

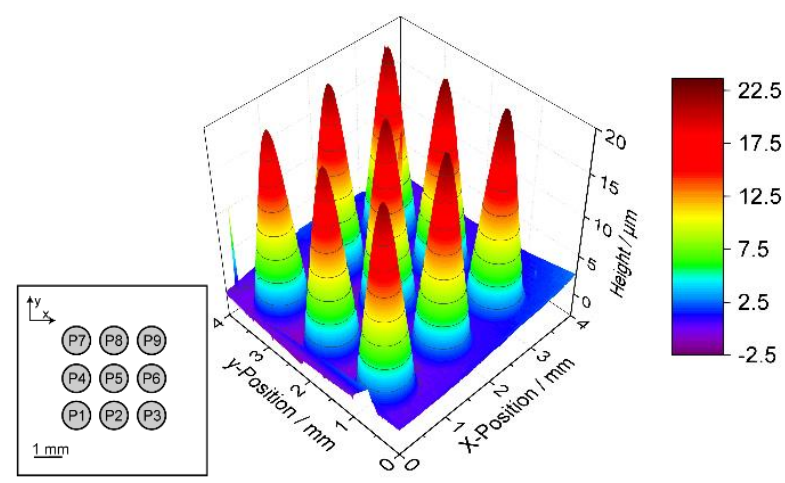

Fig. 2. Measured three-dimensional surface data of a fabricated $174^{\circ}$ axicon array with indications of the lens positions.

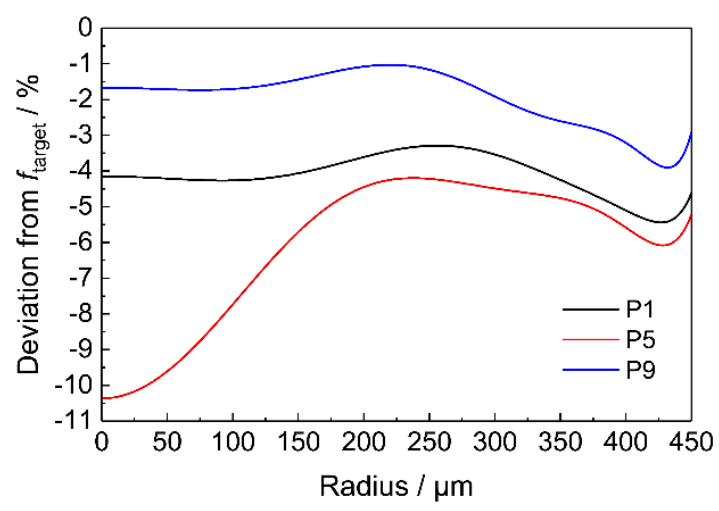

Fig. 3. Deviation of the measured surface data from the simulated target function for three different measured positions.

\section{Conclusions}

We demonstrate a new rapid-prototyping process, which can produce lenses of almost arbitrary shapes. Combing precise FEM simulations with laser-structuring using only a marking laser and a final molding process, we were able to fabricate a $174^{\circ}$ axicon array with a low mean rms surface deviation of $113 \pm 13 \mathrm{~nm}$. This value is comparable with other state-of-the-art technologies for the production of microlenses [5]. In addition, we were able to generate a Bessel beam profile, which showed only small wavefront errors in the range of $\lambda / 6$, indicating high optical quality and the required high rotational symmetry. Our new rapid prototyping process therefore permits the optically precise production of strongly aspherical optics in the $\mathrm{mm}$ range and is also array-compatible.

Tab 1. Measured rms surface deviation at three different lens positions.

\begin{tabular}{|l|l|}
\hline Lens position & Surface error \\
\hline P1 & $99 \mathrm{~nm}$ \\
\hline P5 & $118 \mathrm{~nm}$ \\
\hline P9 & $123 \mathrm{~nm}$ \\
\hline Mean value & $113 \mathrm{~nm}$ \\
\hline Standard deviation & $\pm 13 \mathrm{~nm}$ \\
\hline
\end{tabular}
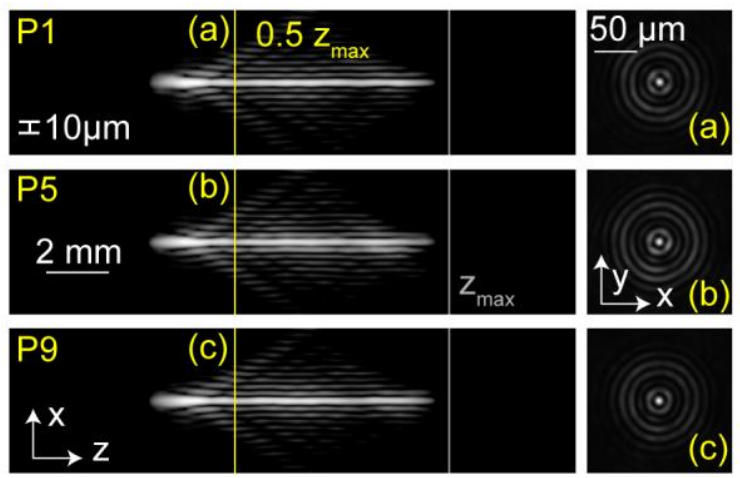

Fig. 4. Measured intensity profile of the Bessel beam (logarithmically scaled) for three different positions (left) with cross-sections at $Z_{\max } / 2$ (right).

This work was supported by the BrainLinks-BrainTools Cluster of Excellence funded by the German Research Foundation (DFG, grant no. EXC 1086).

\section{References}

1. Y. Hu, Y. Xiong, X. Chen, H. Bai, Y. Tian, G. Liu, App. Opt., 57, 15, pp. 4277-4282 (2018)

2. J. Brunne, U. Wallrabe, Opt. Letters, 38, 11 pp. 19391941 (2013)

3. A. Müller, M.C. Wapler, U. Wallrabe, Soft Matter, 15, 4 pp. 779-784 (2019)

4. A. Müller, M.C. Wapler, U.T. Schwarz, M. Reisacher, K. Holc, O. Ambacher, U. Wallrabe, Opt. Express, 24,15 pp. 17433-17452 (2016)

5. H. Ottevaere et al., J. Opt. A, 8, 7 pp. 407-429 (2006) 